\title{
Multi-Purpose Fire Fighting Robotic Vehicle using Android
}

\author{
Mary Agnal P.A ${ }^{1}$, Athira.A ${ }^{2}$, Sanal Kumar T.S \\ B. Tech, Student, Department of Computer Science and Engineering, \\ Universal Engineering College, Vallivattom, Kerala, India ${ }^{1,2}$ \\ Assistant Professor, Department of Computer Science and Engineering, \\ Universal Engineering College, Vallivattom, Kerala, India ${ }^{3}$
}

\begin{abstract}
The fire and rescue robot is an embedded system used to help the rescue team in disaster environment to detect living things and to know the status inside the area to take proper precaution. This robot detects poisonous gas, amount of temperature and motion inside the room. This robot has a roller wheel to climb the stairs and obstacles and also have a 360 degree rotating thermal imaging fire water proof camera for covering huge area. These components are attached with a android device it will send the information to remote android application through wi-fi. The remote application used to control the robot by rescue team
\end{abstract}

Keywords: Embedded System, 360 Degree Rotating Thermal Imaging Camera , Wi-Fi, Rescue

\section{INTRODUCTION}

The system proposed in this paper integrates the use of affordable instruments, connectivity and wireless communication. The proposed fire and rescue robot that detects the poisonous gas the amount of temperature inside the room also detects the motion inside the room. Here introduced a roller robot that has an ability to climb in stair cases and house hold obstacles. This helps the fire and rescue department for knowing the secure condition for entering to the fired room. This robot can be easily accessed with a remote monitor by an external user. We put this robot inside a fired room and controls the direction using the remote monitor with the help of 360 degree rotating thermal imaging camera. that is Fire Water proof camera. The embedded system sense the amount of temperature, density of poisonous gas and motion inside the room. Then this information and data will send to the android application through wi-fi connection. This application will provide the accurate measurements of the value of the temperature and poisonous gas density if it occurs. When motion is detected, the application will send an emergency alert message to the user. Here use two DC motors (12 v) with a driver IC for the movement of the robot that is controlled by an external controller. Here use three sensors are- temperature sensor (LM35), PIR sensor for motion detection and a MQ3 sensor for detecting poisonous gas. These sensors and other modules in the robot are controlled by PIC 16F8768, all modules are associated with PIC.

\section{RELATED WORK}

In [1] This robot is designed with thermostat to sense temperature and camera used to detect living things who is stuck in the disaster situations using the real time video transmission. The aim of the system is directing people among debris, this robot can lift a weight of 200 to 300 grams. Here we use viola jones algorithm for face detection. The GPS is send a data to PC. In the system it gives more priority to detect the location we where the robot send via Bluetooth from sender to receiver and it is controlled by MC and driven via the feedback of the real time camera into picture but the system can get the situation with in hours which can aid in giving priority to people alive ,it is fast, cost efficient and easy to use the use of heavy machinery is prohibited because they would describe the structure, risk in the lights of rescue and victims buried in the rubble. Only by hand should pulverized concrete, glass, furniture and other debris be removed. Rescue specialist used trained search $\operatorname{dog}$ s, camera and listening devices to search for victims from above ground though the search dogs are effecting in finding human underground, they are enables to provide a general description of physical environment the victims locates

In [2] this system is used video surveillance so it also requires a static camera and working is same as CCTV surveillance system so there is no additional expenses on conventional fire sensors. Here fire detection is possible by identify the features of the fire. smoke colour, motion shape and that is powered with fuzzy logic for more accuracy so it will reduce the fault assumptions. Here compare these fire features and generate fire alarm 
In [3] This system consisting transmitter and receiver. The transmitter with smoke sensor, flame sensor and it is guided by ultrasonic sensor mounted on receiver(robot) to avoid obstacles on path here a motor is used for locomotion and a pump is used to sprinkle the wate. The tank can contain water up to 4L. The system pump out the water when it detects any fire features it contains a LED cluster used to know where the fire mishap has occurred the corresponding LED glows when there is fire in that area for which the LED associated

In [4] This robot is powered by solar it is used to detect alive people in disaster situation using a camera installed inside it and it is controlled by rescue the remote of control contains the directions to control this vehicle. the communication between the remote and robot via zigbee module. it also detects fire and it contains battery to charge the robot by using electric current also it helps to work even in the absence of charge by solar.

In[5] This robot is used to detect alive human body in disaster environment by using set of sensors and low cost camera which helpful for rescue operations. This is a mobile robot works in disaster conditions and here a PIR sensor for detect motion of the body and IR sensor to detect obstacles on the way of the robot and temperature sensor to find the amount of temperature. This sensors move in all directions to convert physical quantities like radiation from human body if any character of living detected, then it produce a buzzer and also capture a radio scene using camera. It also send the information about current status and location. RF technology for control the robot, the receiver consist of PC it communicates with robot via RF communication

In[6] This is a fire detection system it contains number of devices working together to detect and warn people through visual and audio appliances when smoke, fire, carbon monoxide or other emergencies are present Development of automatic and intelligent video surveillance systems has been the core trend in the security and guard service industry By observing and utilizing features of fire event, a fast and exact detection process is developed for early fire warning purpose if a fire is detected, a notification is sent through GCM to a custom app along with a URL of the webpage containing images of fire captured by the installed camera system will display the picture of the room state in the web page fire alarm system is real time monitoring system that detects the presence of fire and captures images via a camera installed inside the room when a fire occurs The key feature of the system is the ability to remotely send an alert when a fire is detected The embedded system used to develop this fire alarm system is Raspberry pi, Through this system we get only the picture of the room or the area not get the information about the temperature inside it

In[7] This robot is reprogrammable, multifunction, manipulator designed to move material ,parts, tools, or special device through various programmed motion it is automatically sense the fire used by sensor. This robot consisting of motor, caster wheel, micro controller it consist the robot using some programs fire sensor sense the fire and gives signals to the micro controller RF module helps to control the robot to user from a distance

[8] This system consists of digital packet communication system digital packet communication system transfer data packets for control the fire fighting robot and micro controller status. Remote operator can see the video of the area around the robot through an analog image communication system survives I high temperature

[9] The fire fighting robot controlled by wireless frequency broadcast from remote computer it is very low cost because for building this robot they used recycled parts and materials and also modified electronic devices. This robot consist an arm have 3deree of freedoms one cartesian and two for rotation this gives a full coverage for the water hose in whole directions it is also draw a map separation of fixed area find the living thing is trapped inside it through interface camera

[10] The design and implementation of this fire fighting robot is by using web server. It is used to do the task that is difficult by humans. There is two dc motors used for the movement of this robot and use temperature sensor to know the amount of increasing temperature, gas sensor to find the presence of smoke and a IR sensor to find motion if any living things occur and it is implemented with a DC water pump to extinguish the fire. They are capable of performing repetitive tasks more quickly, cheaply and accurately than humans. The fire fighting robot is design to search for a fire in a small floor plan house of the specific dimension. Mobile is attached to the head of the robot to provide video from the fire prone area. The robot also has the capability to avoid obstacles and upon detection of fire, it forwards a message to a predefined number

In[11] Here a robot which is a cross platform connection between robot and PC. The robot is designed with thermostat to sense temperature, camera to detect people in the rubbles and wheels for the movement with end effector which can lift a weight of around 200 to $300 \mathrm{gm}$. This robot will move in the debris to detect human by using Viola-Jones face detection algorithm. Totally the system aims in directing people among debris, which usually takes a day or so to regular resources into picture but this system can get the situation within hours which can aid in giving priority to people alive, it is fast, cost efficient and easy to use the use of heavy machinery is prohibited because they would 
describe the structure, risking the lives of rescuers and victims buried in the rubble. Only by hand should the pulverized concrete, glass, furniture and other debris be removed. Rescue specialists use trained search dogs, camera and listening devices to search for victims from above ground. though the search dogs are effective in finding human underground, they are unable to provide a general description of physical environment the victim locates

$\operatorname{In}[12]$ this is a fire detection system is number of devices working together to detect and warn people through visual and audio appliances when smoke, fire , carbon monoxide or other emergencies are present Development of automatic and intelligent video surveillance systems has been the core trend in the security and guard service industry By observing and utilizing features of fire event, a fast and exact detection process is developed for early fire warning purpose if a fire is detected, a notification is sent through GCM to a custom app along with a URL of the webpage containing images of fire captured by the installed camera system will display the picture of the room state in the web page fire alarm system is real time monitoring system that detects the presence of fire and captures images via a camera installed inside the room when a fire occurs The key feature of the system is the ability to remotely send an alert when a fire is detected The embedded system used to develop this fire alarm system is Raspberry pi, Through this system we get only the picture of the room or the area not get the information about the temperature inside it

In[13] fire detection system is number of devices working together to detect and warn people through visual and audio appliances when smoke, fire, carbon monoxide or other emergencies are present Development of automatic and intelligent video surveillance systems has been the core trend in the security and guard service industry By observing and utilizing features of fire event, a fast and exact detection process is developed for early fire warning purpose if a fire is detected, a notification is sent through GCM to a custom app along with a URL of the webpage containing images of fire captured by the installed camera system will display the picture of the room state in the web page The fire alarm system is real time monitoring system that detects the presence of fire and captures images via a camera installed inside the room when a fire occurs The key feature of the system is the ability to remotely send an alert when a fire is detected The embedded system used to develop this fire alarm system is Raspberry pi, Through this system we get only the picture of the room or the area not get the information about the temperature inside the room

In[14] This is an unmanned fire extinguishing robot by this purpose, an attempt was made to develop a mobile robot in order to detect fires. That could occur in a closed environment. Here A rotor motor is used for motion beyond barriers by the sensor MZ80, and also use a flame sensor to sense flame and use a fan to extinguish fire. By using the microcontroller module on it evaluates the data in the direction of the software and performs obstacle detection, flame detection, actuation, informing and extinguishing processes. Robot design and application process about the design and development of the mechanical system, the design and development of the electronic system, and the preparation of the necessary software. During the design and development of the mechanical system; Draft drawings, measurements, computer aided design and solid modeling programs. The robot designed in the study was able to detect fire sources randomly placed in random obstacle areas and extinguished with determined fire extinguishing systems.

In[15]this robots can be made which follow the instruction of human operator and perform the task. In this way decisions are taken according to the working conditions by the operator and the task is performed by the robots. Thus, we can use these robots to perform those tasks that may be harmful for humans. This project describes about the DTMF controlled robot which can be controlled to function as a fire extinguisher. It consists of mainly two parts, one is transmitter part and another is receiver part. Here RF transceiver for communication between the devices. The transmitter will transmit the signal and the receiver will receive the signal and make the robot move in respective direction. Here, the program is designed by using Embedded $\mathrm{C}$ programming.

In[16] Robot will detect fire remotely. These robots are mostly useful in industries. The proposed vehicle is able to detect presence of fire and extinguishing it automatically by using temperature sensor. The proposed robot has a water spray which is capable of sprinkling water in 1800 angle. The sprinkler can be move towards the required direction. At the time of moving towards the source of fire it may happen that it will come across some obstacles, then it has obstacle avoiding capability. It detects obstacles using ultrasonic sensors. Communication between the mobile phone and robot will take place through Bluetooth, which will have GUI to control the movement of robot. When mobile get connected to Bluetooth firstly it will set module name, baud rate. It is feasible to implement Bluetooth communication between smartphones and micro-controller. Android controlled robot can be used easily in everyday life such as in homes, market, companies etc. The development of apps for Android in Android SDK is easy and free of cost.

In [17] It is automated fire fighting robot which deals with the fire problems in household, laboratories, small scale industries. This robot follows the predefined path and performs the various tasks. We can use this robot to perform those tasks that may be harmful and dangerous to human. The Fire sensors equipped fixed in the rooms where there will be a possibility of fire is to be occurred. When it senses fire the address of location received by robot and accordingly it will reach to the destination and extinguish fire immediately. 
Vol. 8, Issue 4, April 2019

In [18] This robot is used to find and fight fire remotely through RF communication. The language input allows user to interact with the robot which is familiar to most of the people. The language input allows a user to interact with the robot which is familiar to most of the people. The medium of interaction between humans and computers is on the processing of speech. The proposed vehicle has a water jet spray which is capable of sprinkling water. The sprinkler can be moved towards the required direction. The advent of new high-speed technology provided realistic opportunity for new robot controls and realization of new methods of control theory. This technical improvement together with the need for high performance robots created faster, more accurate and more intelligent robots using new robots control devices, new drivers and advanced

\section{COMPARISON STUDY}

Robotics is the emerging solution to protect human lives and their wealth and surroundings. in existing systems mainly uses conventional fire fighting with the help of fire mans, they pump water to the fired area. Some modern countries using the robots it can even act as a path guider using thermal imaging cameras. Some of other robots will have a pump and small water container with their body to cool its own body when it enters to the fired places, but it is not possible in every condition when the water container is empty, the temperature will destroy the robot and it is water contain capacity is limited. Another one which will not detect any values but when fire is growing up it will pump the water to the fire like a fireman. It has high temperature resistance body so it can be survived in high temperature. But all the existing systems will not discuss the multiple use of same fire fighting robot and they will not think about the use of IOT in this area. Currently internet will be used every one in anywhere but in this area internet will not get any step. And all these above mentioned robots are single user robots and they are getting more time to detect the correct path or guidelines to the fireman

\section{PROPOSED SYSTEM}

\section{A. Design of the Proposed system:}

Block diagram: The block diagram will consist of these 7parts. The temperature sensor, LM3 and motion sensor PIR and the gas sensor MQ3 are connected to a PIC micro controller and the data of sensors and send via a wi-fi module to an android application

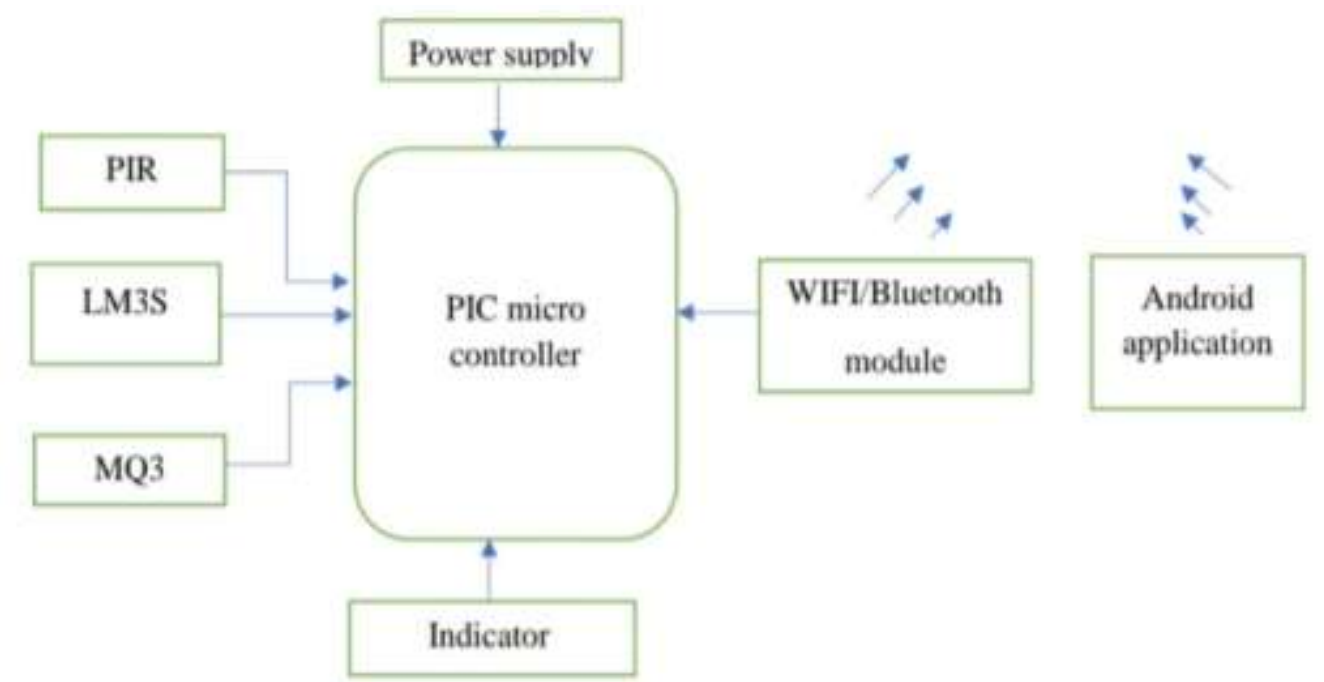

Fig: Block Diagram

Flow Diagram : If the connection is established between the robot and user smartphone then the user can control the movement of device and also get the information given by sensor and camera. The user initially set a temperature value if the value is lesser than of the temperature inside it and no poisonous gas is identified then it will send a secure condition for user otherwise the condition is unsecured. If any motion is detected then the application shows a pop-up message 
International Journal of Advanced Research in Computer and Communication Engineering

Vol. 8, Issue 4, April 2019

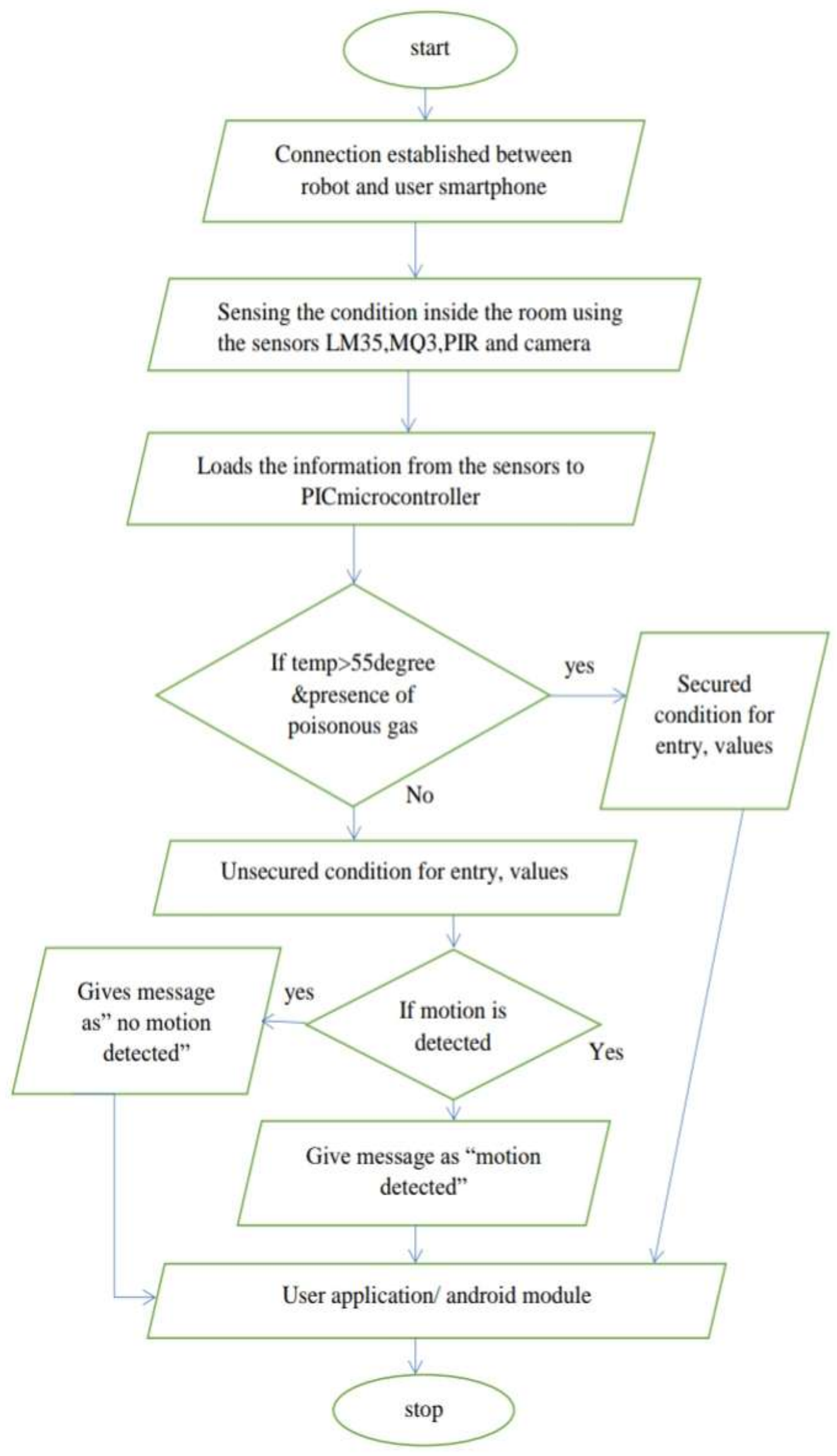

Fig: Flow Diagram 
PIR sensor (motion): A passive infrared sensor (PIR sensor) is an electronic sensor that measures infrared (IR) light radiating from objects in its field of view, they are most often used in PIR based motion detectors. All objects with a temperature above absolute zero emit heat energy in the form of radiation. Usually this radiation isn't visible to the human eye because it radiates at infrared wavelengths, but it can be detected by electronic devices designed for such a purpose. The term passive in this instance refers to the fact that PIR devices do not generate or radiate energy for detection purposes. They work entirely by detecting infrared radiation emitted by or reflected from objects. They do not detect or measure "heat".

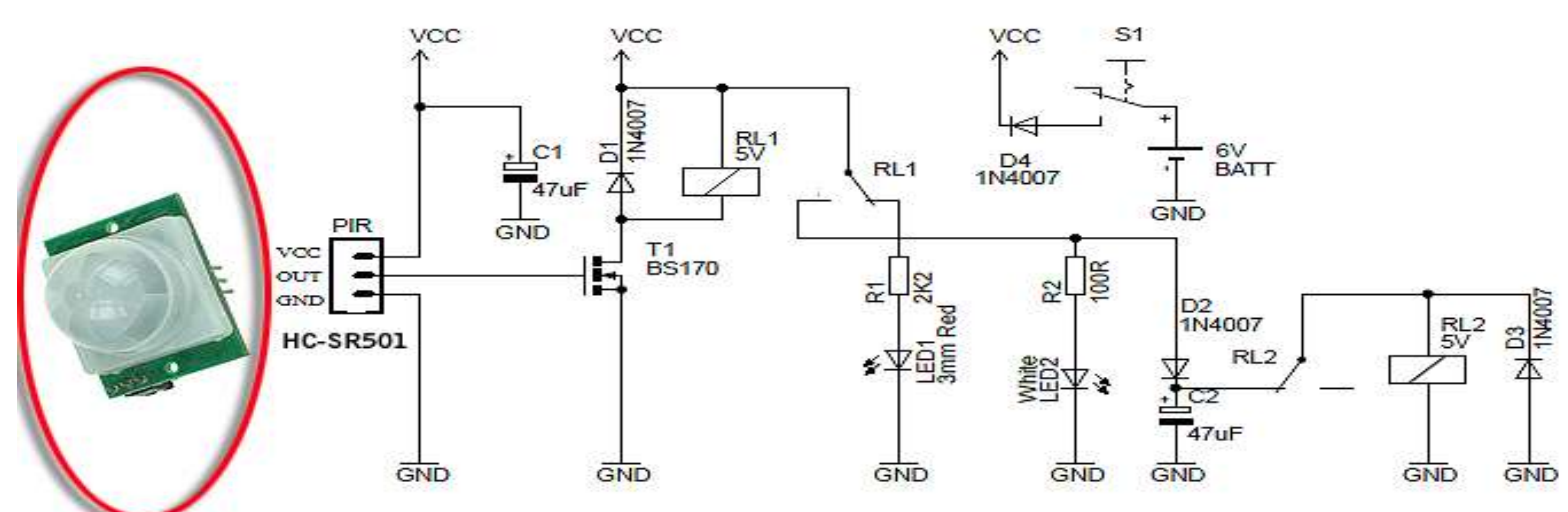

Fig: Pin diagram of PIR sensor

MQ3 sensor (gas): The grove- gas sensor(MQ3) module is useful for gas leakage detection (in home and industry).it is suitable for detecting alcohol, benzine, $\mathrm{CH} 4$, hexane, LPG, CO. due to its sensitivity and fast response time, measurements can be taken as soon as possible. The sensitivity of the sensor can be adjusted by using the potentiometer. The output voltage from the gas sensor increases when the concentration of gas increases. Sensitivity can be adjusted by varying the potentiometer. "The best preheat time for the sensor is above 24 hours".

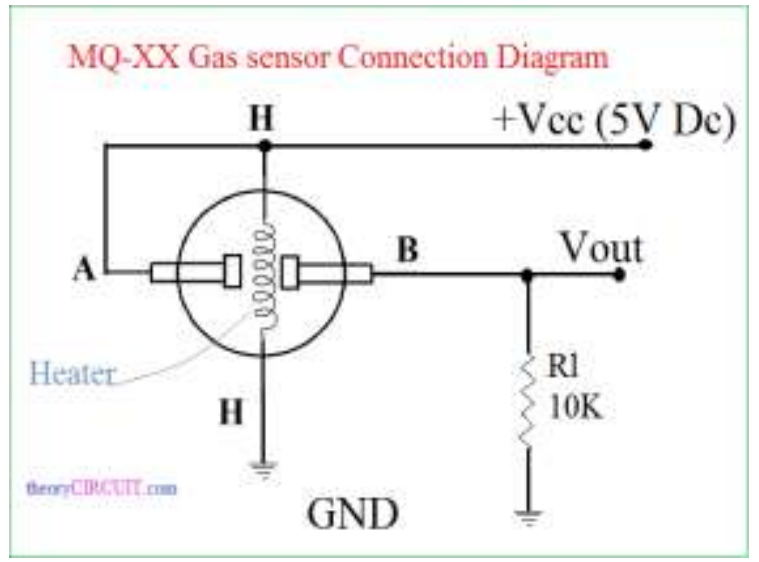

Fig: Pin diagram of MQ3 sensor

PIC16F876 Micro controller: This is powerful (200 nanosecond instruction execution) yet easy-to-program (only 35 single word instructions) CMOS FLASH-based 8-bit microcontroller packs Microchip's powerful PIC architecture into an 28-pin package and is upwards compatible with the PIC16C5X, PIC12CXXX and PIC16C7X devices. PIC16F876 features 256 bytes of EEPROM data memory, self programming, an ICD, 5 channels of 10-bit Analog-to-Digital (A/D) converter, 2 additional timers, 2 capture/compare/PWM functions, the synchronous serial port can be configured as either 3-wire Serial Peripheral Interface (SPITM) or the 2-wire Inter-Integrated Circuit ( $\mathrm{I}^{2} \mathrm{C}^{\mathrm{TM}}$ ) bus and a Universal Asynchronous Receiver Transmitter (USART). All of these features make it ideal for more advanced level A/D applications in automotive, industrial, appliances and consumer applications. 
Vol. 8, Issue 4, April 2019

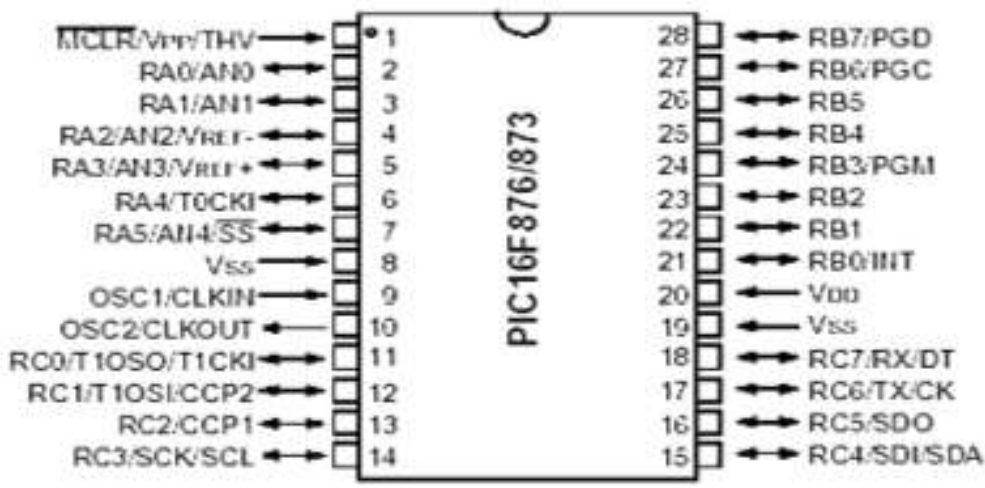

Fig: pin diagram of PIC16f874A

LM35 sensor (temperature): The LM35 series are precision integrated circuit. Temperature devices with an output voltage linearly- proportional to the centigrade temperature. The LM35 device is rated to operate over a $-55 \mathrm{degree} \mathrm{C}$ to 150degree $\mathrm{C}$ temperature range. While the LM3S5C device is rated for a -40degree $\mathrm{C}$ to 110degree $\mathrm{C}$ (-10degree with improved accuracy). It is a Semiconductor based sensor. LM35 temperature sensor is basically a very low cost and easily available sensor. It also an integrated analog temperature sensor. LM35 sensor does not require any external calibration or trimming to provide typical accuracies. The LM35's low output impedance, linear output, and precise inherent calibration make interfacing to readout or control circuitry especially easy.

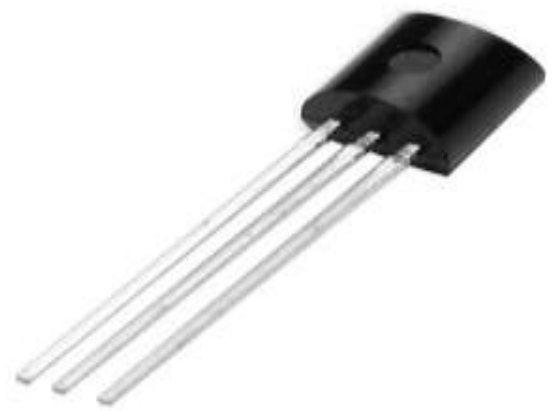

Fig: LM35 sensor

Power supply: A power supply is an electrical device that supplies electric power to an electrical load. The primary function of a power supply is to convert electric current from a source to the correct voltage, current, and frequency to power the load. As a result, power supplies are sometimes referred to as electric power converters. Some power supplies are separate standalone pieces of equipment, while others are built into the load appliances that they power. Other functions that power supplies may perform include limiting the current drawn by the load to safe levels, shutting off the current in the event of an electrical fault, power conditioning to prevent electronic noise or voltage surges on the input from reaching the load, power factor correction, and storing energy so it can continue to power the load in the event of a temporary interruption in the source power

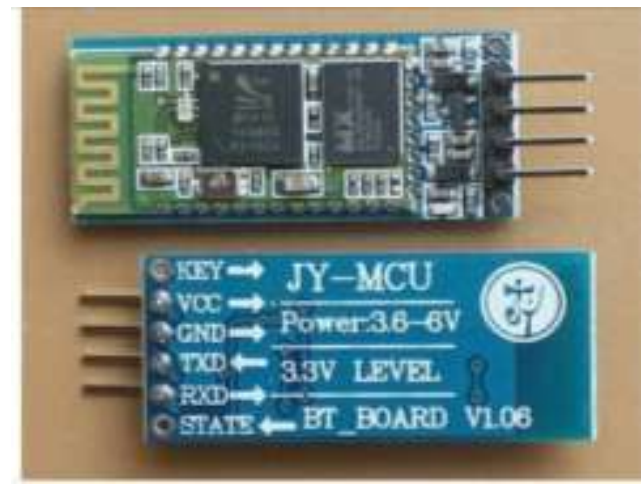

Fig: HC06 bluetooth module

HC06 Bluetooth module: This Bluetooth module can easily achieve serial wireless data transmission. Its operating frequency is among the most popular $2.4 \mathrm{GHz}$ ISM frequency band (i.e. Industrial, scientific and medical). It adopts 
Bluetooth 2.0+EDR standard. In Bluetooth 2.0, signal transmit time of different devices stands at a 0.5 seconds interval so that the workload of bluetooth chip can be reduced substantially and more sleeping time can be saved for bluetooth. This module is set with serial interface, which is easy to use and simplifies the overall design/development cycle

DC Motor: DC motor is any of a class of rotary electrical machines that converts direct current electrical energy into mechanical energy. The most common types rely on the forces produced by magnetic fields. Nearly all types of DC motors have some internal mechanism, either electromechanical or electronic, to periodically change the direction of current flow in part of the motor. DC motors were the first form of motor widely used, as they could be powered from existing direct-current lighting power distribution systems. A DC motor's speed can be controlled over a wide range, using either a variable supply voltage or by changing the strength of current in its field windings. Small DC motors are used in tools, toys, and appliances. The universal motor can operate on direct current but is a motor used for portable power tools and appliances. Larger DC motors are currently used in propulsion of electric vehicles, elevator and hoists, and in drives for steel rolling mills. The advent of power electronics has made replacement of DC motors with AC motors possible in many applications.

L293D IC: The L293 and L293D devices are quadruple high-current half-H drivers. The L293 is designed to provide bidirectional drive currents of up to $1 \mathrm{~A}$ at voltages from $4.5 \mathrm{~V}$ to $36 \mathrm{~V}$. The L293D is designed to provide bidirectional drive currents of up to $600-\mathrm{mA}$ at voltages from $4.5 \mathrm{~V}$ to $36 \mathrm{~V}$. Both devices are designed to drive inductive loads such as relays, solenoids, DC and bipolar stepping motors, as well as other high-current/high-voltage loads in positivesupply applications.

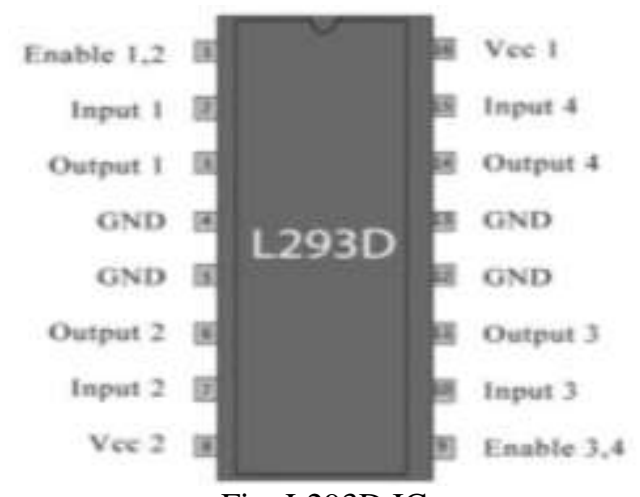

Fig: L293D IC

Thermal Imaging camera: thermal vision technology has a huge advantage over conventional night vision equipment and does not require any possible light to operate, that is this device does not need Infra Red emitter so we can observe everything

Indicators: Indicators are used to represent the critical conditions for alerting the fire mans. Some sort of indicators are LED lights, sound alarm's etc. these alarms are helps to giving attention to the fire man and the other people.

\section{B. Description of the Proposed system:}

Aim of the proposed fire and rescue robot is to minimize the risk of rescue team. It detects the poisonous gas, the amount of temperature inside the room and also detects the motion inside the room. We are introduced a roller robot that have an ability to climb in staircases and household obstacles and it also contain a 360degree rotating thermal imaging fire water proof camera, it can capture images even inside fire and it will not damage in the presence of fire and water. This helps the fire and rescue department for knowing the secure condition for entering to the fired room. This robot can be easily accessed with a remote monitor by an external user. We put this robot inside a fired room and controlled the direction using the remote monitor with the help of a fire resistance camera. The embedded system senses the amount of temperature, density of poisonous gas and the motions inside the room. Then this information and data will send to the android application. This application will provide the accurate measurements of the values of temperature and poisonous gas density if it occurs. When motion is detected the application will send an emergency alert message to the user. Connection between the embedded system/robot and the android application done by using WI-FI / Bluetooth so it can be accessed by multiple users and the camera will provide the visions inside the room, the fire man can see the conditions inside the room it will surely help them for easily finding the location of trapped people inside the room. 


\title{
IJARCCE
}

\author{
Vol. 8, Issue 4, April 2019
}

\section{IMPLEMENTATION}

The implementation of proposed system is done at both hardware and software parts to obtained the system both parts are worked internally connected and worked together. This rescue robot comes equipped with PIR sensor, gas sensor and a temperature sensor to measure the values of the parameters that is send to the rescue operator through android remote application via wi-fi connection and the communication between the microcontroller and android device through Bluetooth. Both connection used socket algorithm for each, to know about the condition and it will reduce the risk to enter a disaster affected area by human. the PIR sensor is used to identify the motion inside the area and give an alert through a message to the android application if any obstacles present on it's path and if the robot need to climb any height, it is possible by using roller. Android technology is used to develop the application it's front end is used by java and xml and the PIC is programmed with Embedded C(assembly language) The rescue can operate and drive this robot using the camera installed inside the robot, it is smoke resistant camera so there is so it can provide a quality video even in the presence of fire and it is 360 degree rotating camera so it can cover a huge area the compact size of robot is ensures the easy carrying .

\section{SIMULATION RESULTS}

The simulation studies involve the deterministic connection between the android app and the embedded system as shown in Fig:1. Here using two socket algorithm for establish the connection between the embedded system, android device and the remote application. There are 2 application used for the connection establishment for controlling and monitoring. All the sensors are connected to the micro controller. The micro controller 16F876 will provide the sensor's value through the Bluetooth module implemented inside it by Bluetooth socketing algorithm and the communication between the two application is done by using wi-fi socketing algorithm. The remote application provide the video surveillance and it also shows the values of the software and it have a refresh button to clear if any connection problem occurred that is shown in Fig:2. The connection between the remote and device application is done by using their IP address and a wi-fi network.

Here 2 DC motors used for the movement of robot with a driver IC L293D and the embedded device consist with sensors and camera inside the android device and a Bluetooth module for the communication between the device application and microcontroller. The whole embedded system is shown in Fig.3. the video send from device app will shown in the remote android device that is shown in fig. 4

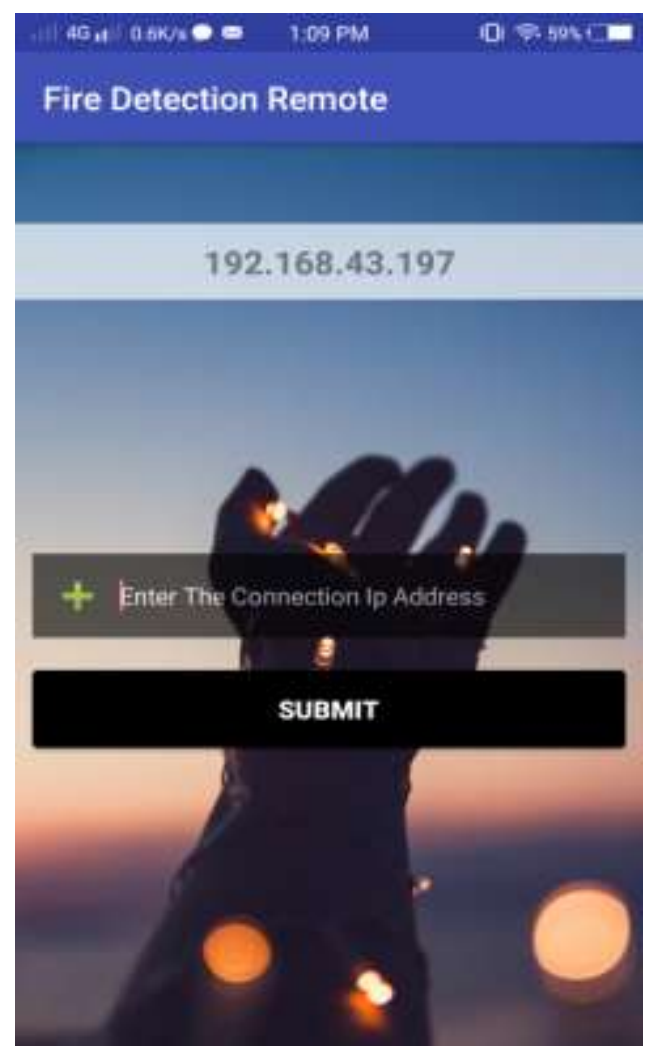

Fig 1: connect the two android devices using its IP address 
Vol. 8, Issue 4, April 2019

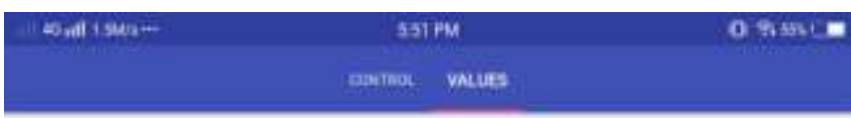

Temperature: 29.30

Motion : Dectected

Gas: $6.25 \%$

Fig: values shown in remote application

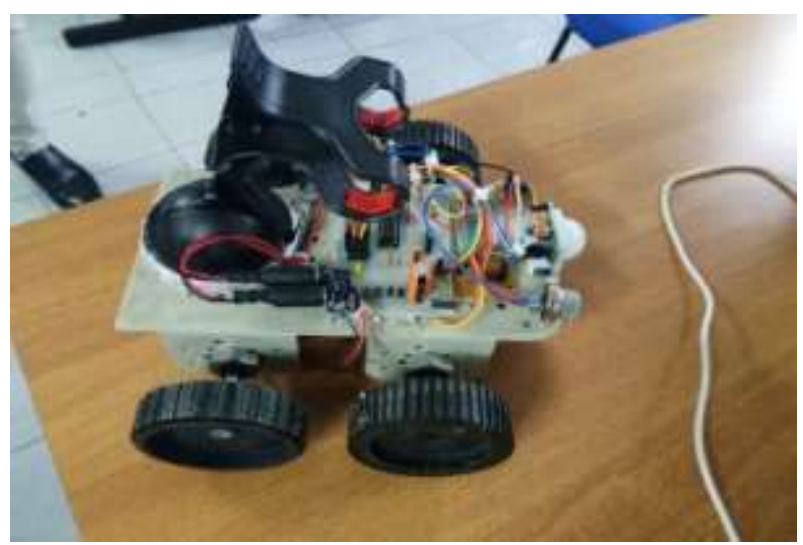

Fig.3: The embedded device

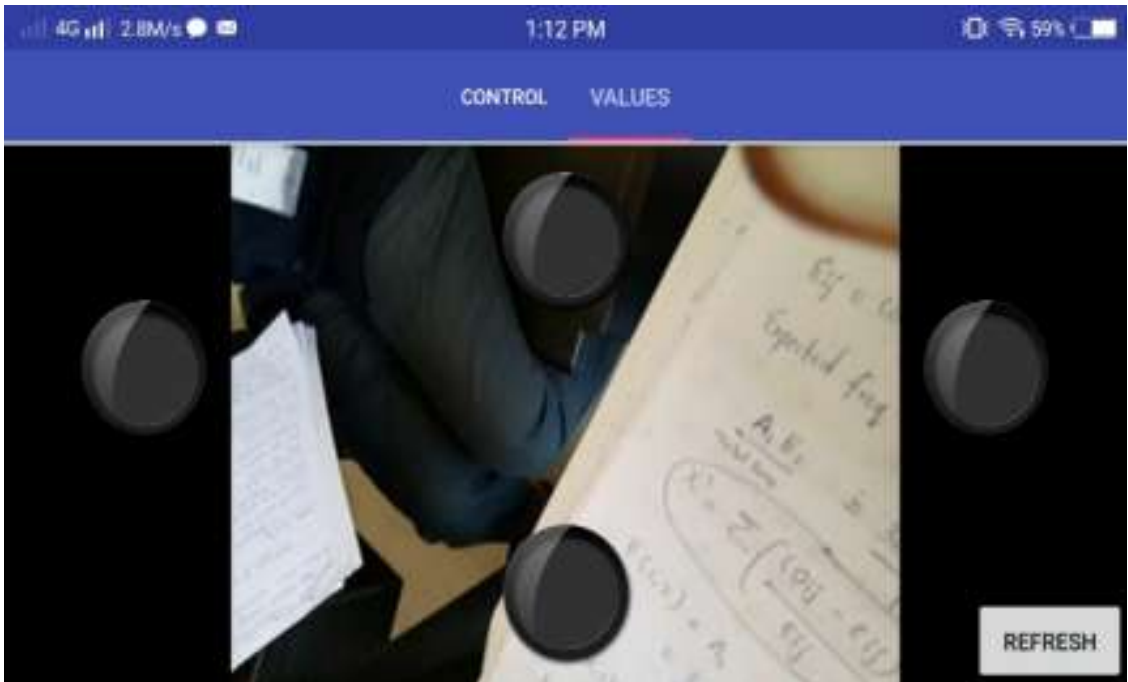

Fig.4: Remote application with video monitoring

VII. CONCLUSION AND FUTURE WORK

\section{CONCLUSION}

The proposed system will help to the fire and rescue team to easily rescue the people. The system is less expensive that the other robot in this field. It will accurately detect the values and every time it will be updated so the fireman can updated the condition inside the room. When any living things trapped inside the room the motion sensor sense it and give an emergency alert message. So the fire man can easily reach the people and time dealy will not take place.

\section{FUTURE WORK}

This embedded system used to help rescue to detect alive human in disaster condition and know about the condition of the affected area is become more effective and widespread because nowadays security is become more important with developments. Through this system we can assure the safety of both rescue and the people inside the disaster area so this system can be used by the safety managers in large buildings and they can handle this easily without any additional 
training and also used for military applications. It can used in many working fields related to chemical and fire it may helps the employees and provide safety for them. We can add more features to this system with new technologies like IoT then the receivers can monitor the area from anywhere

\section{REFERENCES}

[1]. http: //www.ijiet /rescue robot/ISSN: 2319-1058/Vol.2 Issue:3 June 2013

[2]. http://www.ijsr.net/A Methodological survey for Fire detection in camera Surveillance /ISSN:2319-7064 Vol.5, Issue 1, January2016

[3]. http://www.irjet.net /Automatic Fire Extinguisher Robot ISSN:2395-0056/Vol4, Issue5, May 2017

[4]. http://www.irjet,net / Solar Rescue Robot /e-ISSN:2395-0056/Vol:4, Issue 5, May 2017

[5]. http://www.irjet.net /A new approach for detecting alive human beings in devasting environments using a low cost autonomous robot/ Vol 4, Issue 4, April 2017

[6]. http://arduino .cc/en/Reference/Homepage

[7]. http://www.ijcter.com/ automated fire detection surveillance system /June 2016, Vol 2, Issue 6, June 2016

[8]. Dr. A.R.Kondelwar, Mukesh Kumar “"Designing of smart rescue robotic system " www.irjet

[9]. http://www.irjet /Need for Wireless Fire Detection Systems using IoT, Vol:4, Issue :1, Jan-2017

[10]. http://www.ijritcc.org /Fire Fighting Robot, ISSN:2321-8169, Vol:4, Issue:4

[11]. http://www.irjet.net/fire fighting robot e-ISSN: 2395-0056 Vol:4 , Issue: 6 /June -2017

[12]. http://www.iosrjournals.org /Fire-Extinguishing Robot Design by Using Arduino / IOSR Journal of Computer Engineering (IOSR-JCE) eISSN: 2278-0661,p-ISSN: 2278-8727, Volume 18, Issue 6, Ver. V (Nov.-Dec. 2016), PP 113-119

[13]. DTMF Controlled Fire Fighting Robot / Issue - 2016 International Journal of Engineering Research \& Technology (IJERT), ISSN: 2278-0181 NCETET - 2016 Conference Proceedings

[14]. http://www.irjet.net / Android Controlled Firefighting Robot using Arduino/Volume: 05 Issue: 05 | May 2018 , e-ISSN: $2395-0056$ p-ISSN: 2395-0072 